\title{
Fraudulent Accounting and Other Doping Games
}

\author{
by \\ Aleksander Berentsen And Yvan LengwileR*
}

\begin{abstract}
From a game-theoretic point of view, fraudulent accounting to embellish the financial status of a firm and the use of drugs to enhance performance in sports are very similar. We study the replicator dynamics of such games. We allow for heterogeneous populations, such as highly talented versus more mediocre athletes, or high-quality managers versus less able colleagues. For some parameters we find cyclical dynamics, so we may see waves of doping and clean sport, and cycles of fraudulent and honest accounting. Moreover, in some cases, high-ability players are more likely to commit fraud than low-ability ones. (JEL: C 7, M 4)
\end{abstract}

\section{Introduction}

We study a certain class of games, called doping games. A doping game is similar to a rank-order tournament in that two or more parties are assumed to compete for a prize. Only the winner receives a payoff. Players may come in different types indicating their talent. It would seem natural to assume that highly talented contestants also have a higher probability to win. In a doping game, however, players can enhance their performance with a costly activity, which we will call doping. ${ }^{1}$

Interpreting "doping" literally, the description of such games fits the use of performance-enhancing drugs in sports rather well, and we will indeed couch our discussion of the model in these terms. But, as the title suggests, the game we analyze is amenable to interpretations outside of sports. Think, for instance, of a contest between two division managers who compete for being promoted to the top management level. The contestants may have different abilities (strong and weak). The contestant whose division performs better will be promoted. The "performanceenhancing drug" may be fraudulent representation of performance: it has a cost (the manager who is caught cheating will be penalized), but also enhances the probability of promotion. As in a sporting contest, there is just one prize (one promotion) to be distributed, so cheating exerts a negative externality on competitors. Stretching the analogy a bit, we may also interpret the game in terms of companies competing

* We thank the anonymous referees for diligent reviews.

1 The classic reference on rank-order tournaments is LAZEAR AND ROSEN [1981]. The literature that followed this seminal contribution typically does not address issues of dynamics, which are at the center of the present paper. 
for investors. The prize that the CEO of the company receives who reports the highest profit and who can convince the public of its bright future is most likely to keep his job and to receive a large bonus. Embellishing the financial status of the firm redirects capital from competing firms to the cheater, so, as before, there is an externality. Cheating is achieved in that case with the help of an auditor. In that sense, Enron and Worldcom may be viewed as cases of doping, and Arthur Andersen was their amphetamine.

Having analyzed doping and fraudulent behavior as equilibrium phenomena, it seems logical to also discuss policy measures against such behavior. In this short paper, however, we only briefly discuss measures that are available for reducing or inhibiting doping, in section 4.2. For a fuller discussion of such issues in the context of doping games, see BERENTSEN [2002] and BERENTSEN, BRÜGGER, AND LÖRTSCHER [2003].

The simplest version of a doping game has two players with equal talent who compete for a prize. Given their equal abilities, each contestant has a fifty-percent chance of winning. By taking some drug (or committing fraud), a contestant can increase his odds of winning at the expense of his clean (or honest) competitor. If both players dope, the odds of winning are again fifty-fifty, but both players also bear the cost of doping. The game has therefore the structure of a prisoners' dilemma. If the costs of doping are not prohibitive, doping is a dominant strategy.

In this paper, we study the replicator dynamics of doping games between differently talented competitors. The underlying game is a so-called bimatrix game. In contrast to games in which all players are symmetric (same set of strategies and same payoffs), bimatrix games are used to analyze situations with different types of agents. A typical bimatrix game is the battle of the sexes, where there is a conflict concerning parental investment between males and females (HOFBAUER AND SIGMUND [1998, pp. 113ff.]); another is the two-population matching game (WEIBULL [1995, pp. 176ff.]). For the most part, we restrict attention to games between just two types of players (weak and strong), but we also discuss an example of contests among three types of players. Interestingly, for some parameters the replicator dynamics is characterized by cycles similar to the cycles observed in matching-pennies games. Thus, we may see cycles of doping and clean sport, and cycles of fraudulent and honest accounting.

\section{The Model}

We consider sports contests where athletes can use performance-enhancing drugs to improve their game. The population of athletes is of two types: weak and strong. The fraction of strong players is $q$ and assumed to be constant over time, that is, we do not consider the evolutionary dynamics of strong and weak players; rather, we are interested in how the use of performance-enhancing drugs within the strong and within the weak population evolves over time. We assume two large but finite populations of individuals, one such population for the weak and one for the 
strong. The players are randomly drawn from the two populations and matched in pairs. ${ }^{2}$ Accordingly, there are three possible match types: two strong players are matched (ss), two weak players are matched $(w w)$, and a strong player is matched with a weak player $(s w)$.

The players have Von Neumann-Morgenstern preferences about winning and losing the competition. The winning player receives utility $U$, and the losing player receives zero utility. For simplicity, we assume that if no drugs are taken, a strong player when matched with a weak player wins the game with certainty, and when matched with another strong player he wins with probability $1 / 2$. Also, a weak player when matched with another weak player wins the game with probability $1 / 2$. When a doped player is matched with a clean player, regardless whether this player is weak or strong, he wins the game with certainty. When two doped players are matched, the winning probabilities are the same as when two clean players are matched. ${ }^{3}$

Doping improves the performance of the players at $\operatorname{cost} k$, where $0<k<U$. The cost $k$ reflects the fact that athletes do not like to cheat: Each athlete prefers winning without using performance-enhancing drugs over winning with their use. However, each athlete also prefers winning with performance-enhancing drugs over losing without them, i.e., $U>U-k>0$. The cost may also represent the expected health cost and the monetary cost of drugs. In the following we normalize $U=1{ }^{4}$

We assume that each individual is, at each instant of time, programmed to one of the pure strategies, that is, either to take performance-enhancing drugs or to stay clean. Let $p_{s}\left(p_{w}\right)$ be the fraction of strong (weak) players that dope. Denote by $A_{s s}$ the payoff matrix for a strong player who is matched with another strong player, by $A_{s w}$ the payoff matrix for a strong player who is matched with a weak player, by $A_{w s}$ the payoff matrix for a weak player who is matched with a strong player, and by $A_{w w}$ the payoff matrix of a weak player who is matched with another weak player. These payoff matrices are

$$
\begin{gathered}
A_{s s}=A_{w w}=\left[\begin{array}{cc}
\frac{1}{2}-k & 1-k \\
0 & \frac{1}{2}
\end{array}\right], \\
A_{s w}=\left[\begin{array}{cc}
1-k & 1-k \\
0 & 1
\end{array}\right], \quad A_{w s}=\left[\begin{array}{cc}
-k & 1-k \\
0 & 0
\end{array}\right] .
\end{gathered}
$$

2 The analysis in a two-player framework is appropriate for many sports (boxing, tennis, martial arts, etc.). It also applies to team sports, such as soccer or football, where the team manager decides whether her team will use performance-enhancing drugs. It may also apply to other sports, such as cycling or downhill skiing, when two clear leaders compete for the first prize and the other competitors have no influence on this decision.

3 These assumptions about how doping affects the winning probabilities are generalized in section 4. They have no bearing on the qualitative features of the equilibria.

4 That is to say, the cost of doping, $k$, is measured in units of the utility the winner receives. None of the properties of the equilibrium are affected by this normalization. The comparative study of equilibrium dynamics with respect to $k$ is thus equivalent to studying a reciprocal change of $U$ while keeping $k$ constant. 
Consider for example the matrix $A_{s s}$. The first entry in the first row is the expected payoff of a strong player who is matched with another strong player when both players dope: he bears the doping cost $k$, and with probability $1 / 2$ he wins the game. The second entry in the first row represents the payoff when the strong player's partner is clean: in this case the player again bears the doping cost $k$, and he wins the game with certainty. The first entry in the second row is the expected payoff of a strong player who is clean when matched with a strong player who dopes. The payoff is zero because a clean player loses the game with certainty. The second entry in the second row is the expected payoff of a strong player who is clean and matched with another strong and clean player. The payoff is $1 / 2$, because the two players are equally likely to win the game. The other payoff matrices have a similar interpretation.

To proceed, denote by $u_{s d}\left(u_{w d}\right)$ the average payoff of strong (weak) players that dope, and by $u_{s c}\left(u_{w c}\right)$ the average payoff of strong players that stay clean. The average payoffs of strong and of weak players are denoted by $u_{s}$ and $u_{w}$, respectively. These average payoffs are calculated as follows:

$$
\begin{aligned}
u_{s d} & =q(1,0) A_{s s}\left(p_{s}, 1-p_{s}\right)^{T}+(1-q)(1,0) A_{s w}\left(p_{w}, 1-p_{w}\right)^{T}, \\
u_{s c} & =q(0,1) A_{s s}\left(p_{s}, 1-p_{s}\right)^{T}+(1-q)(0,1) A_{s w}\left(p_{w}, 1-p_{w}\right)^{T}, \\
u_{s} & =p_{s} u_{s d}+\left(1-p_{s}\right) u_{s c}, \\
u_{w d} & =q(1,0) A_{w s}\left(p_{s}, 1-p_{s}\right)^{T}+(1-q)(1,0) A_{w w}\left(p_{w}, 1-p_{w}\right)^{T}, \\
u_{w c} & =q(0,1) A_{w s}\left(p_{s}, 1-p_{s}\right)^{T}+(1-q)(0,1) A_{w w}\left(p_{w}, 1-p_{w}\right)^{T}, \\
u_{w} & =p_{w} u_{w d}+\left(1-p_{w}\right) u_{w c} .
\end{aligned}
$$

For example, consider the average payoff of a strong player who dopes, $u_{s d}$. This player is matched with another strong player with probability $q$, and with a weak player with probability $1-q$. In the former case the expected payoff is $(1,0) A_{s s}\left(p_{s}, 1-p_{s}\right)^{T}$ because the other strong player is a doper with probability $p_{s}$. In the later case the expected payoff is $(1,0) A_{s w}\left(p_{w}, 1-p_{w}\right)^{T}$, because the weak partner dopes with probability $p_{w}$.

The standard replicator dynamics is given by

$$
\begin{aligned}
& \dot{p}_{s}=p_{s}\left(u_{s d}-u_{s}\right)=\frac{1}{2}\left(1-p_{s}\right) p_{s}\left[-2 k+q+2(1-q) p_{w}\right], \\
& \dot{p}_{w}=p_{w}\left(u_{w d}-u_{w}\right)=\frac{1}{2}\left(1-p_{w}\right) p_{w}\left(1-2 k+q-2 q p_{s}\right) .
\end{aligned}
$$

The idea behind (2) is that the fraction of doped agents of each type increases if doping yields a larger average payoff than staying clean (if $u_{i d}$ is larger than $u_{i c}$, which is equivalent to $u_{i d}>u_{i}$ for $i=s, w$ ); the fraction decreases if staying clean is more profitable. This replicator dynamics goes back to a paper by TAYLOR AND JONKER [1978]. It is today widely used in evolutionary game theory to describe the dynamics of the composition of strategies in a population. ${ }^{5}$ The basic idea is that the share of a strategy that is more successful on average increases over time.

5 Other dynamics have also been investigated. A general approach is put forth by KANDORI, MAILATH, AND ROB [1993]. 
In the following we want to characterize the stationary states. For this purpose denote the state space by $\mathscr{P}=[0,1]^{2}$. Any point $p:=\left(p_{s}, p_{w}\right) \in \mathscr{P}$ represents a distribution of individuals in the two populations $i \in\{s, w\}$, across the pure strategies $d$ and $c$ available to the agents. Inspection of (2) reveals that the strategy profiles $(1,1)$, $(1,0),(0,1)$, and $(0,0)$ are stationary states for all $(k, q) \cdot{ }^{6}$ In addition, for some $(k, q)$ there are also stationary states in the interior of $\mathscr{P}$. Define the set

$$
\diamond:=\{(k, q) \mid \min \{2-2 k, 2 k\}>q>\max \{1-2 k, 2 k-1\}\} .
$$

If $(k, q) \in \diamond$, the point

$$
\left(\hat{p}_{s}, \hat{p}_{w}\right):=\left(\frac{1-2 k+q}{2 q}, \frac{2 k-q}{2(1-q)}\right)
$$

is stationary.

We now characterize the stability properties of the stationary states. Propositions 1 and 2 concern different areas of the parameter space that give rise to different stability properties of the equilibria. It is helpful to define the following sets (see Figure 1, where we have labeled them accordingly):

$$
\begin{aligned}
D D & :=\{(k, q) \mid 1-2 k>q>2 k\}, \\
d D & :=\{(k, q) \mid q<\min \{1-2 k, 2 k\}\}, \\
D c & :=\{(k, q) \mid q>\max \{1-2 k, 2 k\}\}, \\
C C & :=\{(k, q) \mid 2 k-1>q>2(1-k)\}, \\
c C & :=\{(k, q) \mid q<\min \{2 k-1,2(1-k)\}\}, \\
C d & :=\{(k, q) \mid q>\max \{2 k-1,2(1-k)\}\} .
\end{aligned}
$$

The letters refer to the equilibrium pure strategies of the strong and the weak players, respectively. Capital letters indicate that the respective strategy is dominant in this particular region. Lowercase letters indicate that the strategy is only a best reply.

Define further

$$
\begin{array}{ll}
\mathscr{P}_{d d}=\mathscr{P} \backslash\{(0,0),(0,1),(1,0)\}, & \mathscr{P}_{c c}=\mathscr{P} \backslash\{(0,1),(1,0),(1,1)\}, \\
\mathscr{P}_{c d}=\mathscr{P} \backslash\{(0,0),(1,0),(1,1)\}, & \mathscr{P}_{d c}=\mathscr{P} \backslash\{(0,0),(0,1),(1,1)\} .
\end{array}
$$

Proposition 1 (i) If $(k, q) \in D D \cup d D$, any initial state $p \in \mathscr{P}_{d d}$ converges to state (1,1). (ii) If $(k, q) \in C C \cup c C$, any initial state $p \in \mathscr{P}_{c c}$ converges to state $(0,0)$. (iii) If $(k, q) \in D c$, any initial state $p \in \mathscr{P}_{d c}$ converges to state $(1,0)$. (iv) If $(k, q) \in C d$, any initial state $p \in \mathscr{P}_{c d}$ converges to state $(0,1)$.

Proof We only prove statement (i), because the proofs of statements (ii) to (iv) are analogous.

6 If $q=1-2 k$, then all points in $\{(1, z) \mid z \in[0,1]\}$ are also stationary states. Along the same lines, the states in $\{(0, z) \mid z \in[0,1]\}$ are stationary if $q=2 k-1$, the states in $\{(z, 1) \mid z \in[0,1]\}$ are stationary if $q=2(1-k)$, and the states in $\{(0, z) \mid z \in[0,1]\}$ are stationary if $q=2 k$. Throughout the paper we ignore these special cases. 
Stable Points

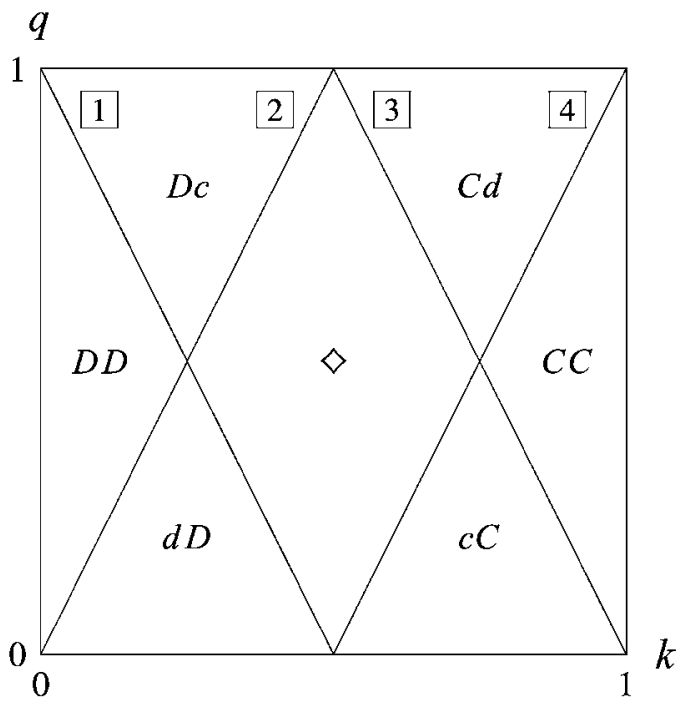

The four lines are defined by...

$1 \quad q=1-2 k$

$2 \quad q=2 k$

$3 q=2-2 k$

$4 q=2 k-1$

Case 1: $(k, q) \in D D=\{(k, q) \mid 1-2 k>q>2 k\}$. From (2) we get

$$
\begin{aligned}
& \dot{p}_{s}=\frac{1}{2}\left(1-p_{s}\right) p_{s}\left[-2 k+q+2(1-q) p_{w}\right]>\left(1-p_{s}\right) p_{s}(1-q) p_{w}>0, \\
& \dot{p}_{w}=\frac{1}{2}\left(1-p_{w}\right) p_{w}\left(1-2 k+q-2 q p_{s}\right)>\left(1-p_{w}\right) p_{w} q\left(1-p_{s}\right)>0 .
\end{aligned}
$$

If $(k, q) \in D D$, doping is a dominant strategy for both player types, so that any initial state $p \in \mathscr{P}_{d d}$ converges to $(1,1)$.

Case 2: $(k, q) \in d D=\{(k, q) \mid q<\min \{1-2 k, 2 k\}\}$. From (2) we get

$$
\begin{aligned}
& \dot{p}_{s}=\frac{1}{2}\left(1-p_{s}\right) p_{s}\left[-2 k+q+2(1-q) p_{w}\right]>0 \text { if } p_{w}>\frac{2 k-q}{2(1-q)}>0, \\
& \dot{p}_{w}=\frac{1}{2}\left(1-p_{w}\right) p_{w}\left(1-2 k+q-2 q p_{s}\right)>\left(1-p_{w}\right) p_{w} q\left(1-p_{s}\right)>0 .
\end{aligned}
$$

In this case the weak players have a dominant strategy to dope, so that eventually all weak players dope. The strong players have no dominant strategy. However, for $p_{w}$ sufficiently large we have $\dot{p}_{s}>0$. Thus, if $(k, q) \in d D$, any $p \in \mathscr{P}_{d d}$ converges to $(1,1)$.

Q.E.D.

Proposition 1 establishes that the stationary point $(1,1)$ is almost globally stable if $(k, q) \in D D \cup d D$. The "almost" refers to the fact that the unstable stationary states $(1,0),(0,1),(0,0)$ are not in the basin of attraction of $(1,1)$. Analogous statements apply to the stationary points in the other regions defined above. They, too, are either almost globally stable or unstable. 
Proposition 2 Assume $(k, q) \in \diamond$. The interior stationary state $\hat{p}$ is Lyapunovstable, but not asymptotically stable. Moreover, for each $p \in$ int $\mathscr{P}$, there is a cyclical orbit $\mathscr{O} \subset$ int $\mathscr{P}$ that is a Lyapunov-stable set.

Proof We first show that $\hat{p}$ is a Lyapunov-stable point. It suffices to show that the system (2) has an associated Lyapunov function for $\hat{p}$ [defined in (4)] with zero time derivative.

Consider the following function:

$$
\begin{gathered}
\mathscr{L}(p):=L(\hat{p})-L(p), \\
\text { with } \quad L(p):=(1-2 k+q) \ln \left(p_{s}\right)+(-1+2 k+q) \ln \left(1-p_{s}\right) \\
+(2-2 k-q) \ln \left(1-p_{w}\right)+(2 k-q) \ln \left(p_{w}\right) .
\end{gathered}
$$

Step 1: $\mathscr{L}$ attains a global minimum at $\hat{p}$. By definition, $\mathscr{L}(\hat{p})=0$. We need to show that $\mathscr{L}(p)>0$ for all $p \neq \hat{p}$. To establish this, note that the Hessian of $\mathscr{L}$,

$$
\left[\begin{array}{cc}
\frac{1-2 k+q}{p_{s}^{2}}+\frac{-1+2 k+q}{\left(1-p_{s}\right)^{2}} & 0 \\
0 & \frac{2-2 k-q}{\left(1-p_{w}\right)^{2}}+\frac{2 k-q}{p_{w}^{2}}
\end{array}\right],
$$

is positive definite everywhere if $(k, q) \in \diamond$. Moreover, the gradient of $\mathscr{L}$ vanishes at $\hat{p}$. Thus, $\mathscr{L}$ attains a global minimum at $\hat{p}$.

Step 2: $\dot{\mathscr{L}}=0$ along a solution path. We have

$$
\dot{\mathscr{L}}(p)=\frac{\partial \mathscr{L}}{\partial p_{s}} \dot{p}_{s}+\frac{\partial \mathscr{L}}{\partial p_{w}} \dot{p}_{w},
$$

where $\dot{p}_{s}$ and $\dot{p}_{w}$ are defined in (2). We want to show that $\dot{\mathscr{L}}(p)=0$ for all $p \in \operatorname{int} \mathscr{P}$ if $(k, q) \in \diamond$. To do so note that

$$
\begin{aligned}
\frac{\partial \mathscr{L}}{\partial p_{s}} \dot{p}_{s}= & -\frac{1}{2}(1-2 k+q)\left(1-p_{s}\right)\left[-2 k+q+2(1-q) p_{w}\right] \\
& +\frac{1}{2}(-1+2 k+q) p_{s}\left[-2 k+q+2(1-q) p_{w}\right], \\
\frac{\partial \mathscr{L}}{\partial p_{w}} \dot{p}_{w}= & \frac{1}{2}(2-2 k-q) p_{w}\left(1-2 k+q-2 q p_{s}\right) \\
& -(2 k-q) \frac{1}{2}\left(1-p_{w}\right)\left(1-2 k+q-2 q p_{s}\right) .
\end{aligned}
$$

Simplifying yields

$$
\frac{\partial \mathscr{L}}{\partial p_{s}} \dot{p}_{s}=-\frac{1}{2}\left(1-2 k+q-2 q p_{s}\right)\left[-2 k+q+2(1-q) p_{w}\right]=-\frac{\partial \mathscr{L}}{\partial p_{w}} \dot{p}_{w} .
$$

Evidently, $\partial \mathscr{L} / \partial p_{s} \dot{p}_{s}+\partial \mathscr{L} / \partial p_{w} \dot{p}_{w}=0$, establishing the claim.

Steps 1 and 2 establish that $\mathscr{L}$ is a Lyapunov function for $\hat{p}$, hence $\hat{p}$ is a Lyapunovstable point. We now show that there are Lyapunov-stable orbits around $\hat{p}$. 
Step 3: For each $\bar{p} \in$ int $\mathscr{P}$ there exists a cyclical orbit $\mathscr{O} \subset$ int $\mathscr{P}$ that is a Lyapunovstable set (WEIBULL [1995, p. 244]). Let $\mathscr{O}$ be the solution orbit associated with some fixed $\left(\bar{p}_{s}, \bar{p}_{w}\right):=\bar{p} \in$ int $\mathscr{P}$. Let $\tilde{p}:=\left(\tilde{p}_{s}, \tilde{p}_{w}\right)$. Formally,

$$
\mathscr{O}:=\left\{\tilde{p} \mid \exists T \tilde{p}=\bar{p}+\int_{0}^{T} \dot{p} d t\right\}
$$

where $\dot{p}$ is given by (2). $\mathscr{O}$ is the collection of all $\tilde{p}$ that the system reaches sometime in the future if we start out from $\bar{p}$. By step 2 we have $\dot{\mathscr{L}}(p)=0$ along any such solution orbit. It therefore follows that $\mathscr{L}(p)=: \lambda_{\mathscr{O}}$ is constant for all $p \in \mathscr{O}$. Define now a Lyapunov function for this orbit as follows:

$$
\mathscr{L}_{\mathscr{O}}(p):=\left(\mathscr{L}(p)-\lambda_{\mathscr{O}}\right)^{2} .
$$

Clearly, $\mathscr{L}_{\mathscr{O}}(p) \geq 0$, with equality if and only if $p \in \mathscr{O}$. Moreover, $\dot{\mathscr{L}}_{\mathscr{O}}(p)=0$; hence $\mathscr{O}$ is a Lyapunov-stable set, but not an asymptotically stable set.

Q.E.D.

The stable cyclical orbits of Proposition 2 are reminiscent of the evolutionary dynamics of the matching-pennies game. This is no coincidence, because in essence the doping game has the same structure. The strong player wants to dope only if the weak player does. For the weak player, however, doping is only interesting if the strong player stays clean. The point $\hat{p}$, which is stationary if $(k, q) \in \diamond$, is equivalent to the mixed Nash equilibrium of the standard matching-pennies game.

The comparative statics of the stationary points are somewhat surprising. Interestingly, the strong and weak players react differently to a change in the cost of doping. Given $q$, the fraction of strong players that use doping decreases monotonically as the cost of doping $k$ increases. The same is not true for the population of weak players. If $q>1 / 2$, all weak players use doping at the stationary point if $k<(1-q) / 2$. On increasing the cost of doping marginally above this threshold, the fraction of weak players using doping in the stationary equilibrium discontinuously drops to zero, as long as $(1-q) / 2<k<q / 2$. On increasing $k$ further we enter the $\diamond$-region. In this region, the fraction of weak players using doping in the interior stationary state $\hat{p}$ increases with the cost of doping. The reason for this counterintuitive comparative statics is that the decrease in the fraction of strong players that use doping makes doping more attractive for weak players. This effect is stronger than the direct cost effect, so that, in sum, the fraction of weak players that use doping increases with the cost of doping in this region.

Note also that if $(k, q) \in \diamond$ and $k<1 / 2$, then $p_{s}$ exceeds $p_{w}$ in the interior strategy stationary point. Thus, it is possible that strong players are more likely to use performance-enhancing drugs than weak players.

\section{Interpretation}

Propositions 1 and 2 are most easy to understand in connection with Figure 1. Proposition 1 establishes that if the doping cost $k$ and the fraction of strong players $q$ 
are in region $D D$ or $d D$, then all agents dope eventually. The intuitive reason for this result is quite clear for region $D D$. In this region, the cost of doping $k$ is sufficiently small so that both types of agents have a dominant strategy to dope. This means that, no matter what the opponent does, doping generates a larger average payoff than staying clean. Clearly, in such a situation, the replicator dynamics causes the doping frequency to increase monotonically over time, until eventually all players dope.

The argument is a little bit more involved for region $d D$. In this region, the fraction of weak players is large. Therefore, any player is most likely to be matched with a weak player. Since doping costs are relatively small, weak players have a dominant strategy to dope. Consequently, the replicator dynamics causes weak types to dope with ever higher frequency, until eventually all of them dope. In contrast, the strong players do not have a dominant strategy in this region. For them, doping is optimal only if sufficiently many weak players dope. But since the evolutionary dynamics leads weak types to dope no matter what, strong players will follow and eventually all of them dope as well.

The logic of region $D c$, which features a large fraction of strong players, is as follows: here, only the strong players have a dominant strategy to dope, because players are most likely to be matched with strong players. Consequently, the replicator dynamics leads strong types to dope with ever higher frequency, until eventually all of them dope. In contrast, for weak players doping is profitable only if strong types stay clean, because weak players cannot win against doped strong players, even if they use doping. Since the probability of being matched with a strong player is high, doping does not pay on average for weak players if strong players dope with sufficiently high probability. As a result, the evolutionary dynamics drives all weak players to stay clean eventually. The intuitive reasons for regions $C C, c C$, and $C d$ are analogous.

Proposition 2 describes the equilibria if the parameters are in region $\diamond$. The key concept here is Lyapunov stability: "Intuitively, a state $p \in \mathscr{P}$ is Lyapunov stable if no small change in the population composition can lead it away, and $p \in \mathscr{P}$ is asymptotically stable if moreover any sufficiently small such change results in a movement back toward $p$ " (WeIBULL [1995, p. 75], notation adapted). Lyapunov stability is weaker than asymptotic stability, because it does not require convergence. An extension of this definition is the concept of a Lyapunov-stable set: consider a closed set $\mathscr{O} \subset \mathscr{P}$. Suppose the dynamics is such that the equilibrium stays within $\mathscr{O}$ if we start from $p \in \mathscr{O}$. We call such a set $\mathscr{O}$ a solution orbit. Suppose further that a small move from $p$ to $p^{\prime}$ leads to a solution orbit $\mathscr{O}^{\prime}$ that is close to $\mathscr{O}$. If this is the case, we say that $\mathscr{O}$ is a Lyapunov-stable set (WEIBULL [1995, p. 244]). Intuitively, a Lyapunov-stable set is an equilibrium with cycles that is not changed a lot by small shocks.

Proposition 2 establishes that the points $\hat{p}$ as defined in (4) are Lyapunov-stable points if the parameters of the game are in the region $\diamond$. Moreover, in this region there are also Lyapunov-stable sets that result in cyclical equilibria, as depicted in Figure 2. From (2) and (4) we can determine that in equilibrium the state cycles 
Figure 2

Orbits for Different Costs (left) and Different Initial States (right)
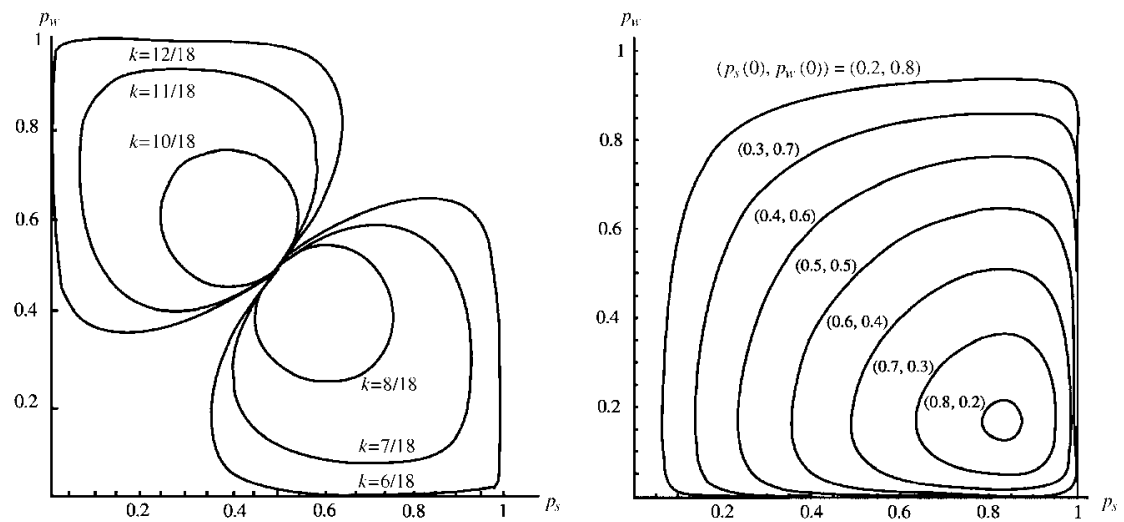

in these orbits in a clockwise direction. This induces cyclical ups and downs of $p_{w}$ and $p_{s}$, but with a phase shift.

\section{Generalizations}

In this section we consider three generalizations. First we consider the case of less than perfectly effective drugs. Then we address the effects of possible detection and punishment of doped players. Finally, we extend the analysis to populations with three different ability levels.

\subsection{Less Effective Drugs}

So far we have assumed that an athlete that takes performance-enhancing drugs wins the game with certainty if his competitor is clean. We now consider less effective drugs, and we also assume that a strong player matched with a weak player wins the game with probability $\sigma$, where $1 \geq \sigma \geq 0.5$. Denote by $\delta \geq 0.5$ the probability that a doped strong (weak) player wins when matched with a clean strong (weak) player. The difference $\delta-0.5$ specifies the effectiveness of performance-enhancing drugs in the sense of measuring the increase of a player's winning probability when only that player dopes. Denote further by $\delta_{s w}\left(\delta_{w s}\right)$ the probability that a doped strong (weak) player wins when matched with a clean weak (strong) player, where $\delta_{s w} \geq \sigma$ $\left(\delta_{w s} \geq 1-\sigma\right)$. Note that in the previous section we have assumed $\sigma=\delta=\delta_{s w}=$ $\delta_{w s}=1$, i.e., we have assumed maximal effectiveness of performance-enhancing drugs. In this section we argue that nothing changes qualitatively if we assume that performance-enhancing drugs are less than completely effective. 
With these new specifications of the winning probabilities the standard replicator dynamics is

(6)

$$
\begin{aligned}
& \dot{p}_{s}=\frac{1}{2}\left(1-p_{s}\right) p_{s} {[-2 k+(2 \delta-1) q} \\
&+\left.2(1-q)\left(\delta_{s w}-\sigma\right)-2(1-q)\left(1+\delta_{s w}-\delta_{w s}-2 \sigma\right) p_{w}\right], \\
& \dot{p}_{w}=\frac{1}{2}\left(1-p_{w}\right) p_{w}[-2 k+(2 \delta-1)(1-q) \\
&\left.+2 q\left(\delta_{w s}+\sigma-1\right)+2 q\left(1+\delta_{s w}-\delta_{w s}-2 \sigma\right) p_{s}\right] .
\end{aligned}
$$

Inspection of (6) reveals that this dynamics has the same qualitative properties as in the previous section. In particular the profiles $(1,1),(1,0),(0,1)$, and $(0,0)$ are stationary states for all $(k, q) \in \mathscr{P}$. Also, for some $(k, q)$ there are interior distributions $\left(\hat{\hat{p}}_{s}, \hat{\hat{p}}_{w}\right)$ that are stationary:

$$
\begin{aligned}
& \hat{\hat{p}}_{s}=\frac{2 k-(2 \delta-1)(1-q)-2 q\left(\delta_{w s}+\sigma-1\right)}{2 q\left(1+\delta_{s w}-\delta_{w s}-2 \sigma\right)}, \\
& \hat{\hat{p}}_{w}=\frac{-2 k+(2 \delta-1) q+2(1-q)\left(\delta_{s w}-\sigma\right)}{2(1-q)\left(1+\delta_{s w}-\delta_{w s}-2 \sigma\right)} .
\end{aligned}
$$

Note that Propositions 1 and 2 continue to hold because (6) has the same qualitative properties as (2). That is to say, all combinations of noninterior strategies are stationary points, but, depending on $(k, q)$, at most one of them is asymptotically stable. For some $(k, q)$, there is an interior stationary point that is Lyapunov-stable, but not asymptotically stable. In that case, there are also Lyapunov-stable cyclical orbits hovering around the stationary point.

\subsection{Detection and Punishments}

We have not addressed the possibility of detection and punishments so far. We add this possibility now as follows. Suppose tests are performed after the contest with probability $\tau$. The test detects a doped player with probability $\beta$, so $1-\beta$ is the probability of false negatives. The test may also produce false positives, that is, erroneously identify clean players as doped, with probability $\alpha$. A perfect test would have $\alpha=0$ and $\beta=1$. The test is at least to some extent informative if $\beta>\alpha$, and this is what we assume. An agent who is identified as doped is subject to a punishment $\pi$.

Let $\tilde{u}_{s d}$ denote the average payoff of strong doped players, and likewise for $\tilde{u}_{s c}, \tilde{u}_{w d}$, $\tilde{u}_{w c}$. The expected punishment of doped players is given by the probability that a test is performed $\tau$ times the probability of being detected $\beta$ times the punishment $\pi$, so $\tilde{u}_{s d}=u_{s d}-\tau \beta \pi$ and $\tilde{u}_{w d}=u_{w d}-\tau \beta \pi$. For clean players, the expected punishment is $\tau \alpha \pi$, so $\tilde{u}_{s c}=u_{s c}-\tau \alpha \pi$ and $\tilde{u}_{w c}=u_{w c}-\tau \alpha \pi$, where $u_{i j}$ are given by (1). As before, $\tilde{u}_{s}:=p_{s} \tilde{u}_{s d}+\left(1-p_{s}\right) \tilde{u}_{s c}$ and $\tilde{u}_{w}:=p_{w} \tilde{u}_{w d}+\left(1-p_{w}\right) \tilde{u}_{w c}$ denote average payoffs of 
strong and weak players, respectively. The replicator dynamics is now

$$
\begin{aligned}
\dot{p}_{s} & =p_{s}\left(\tilde{u}_{s d}-\tilde{u}_{s}\right) \\
& =\frac{1}{2}\left(1-p_{s}\right) p_{s}\left\{-2[k+\tau(\beta-\alpha) \pi]+q+2(1-q) p_{w}\right\}, \\
\dot{p}_{w} & =p_{w}\left(\tilde{u}_{w d}-\tilde{u}_{w}\right) \\
& =\frac{1}{2}\left(1-p_{w}\right) p_{w}\left\{1-2[k+\tau(\beta-\alpha) \pi]+q-2 q p_{s}\right\} .
\end{aligned}
$$

Comparing this with (2) reveals that tests and punishments have precisely the same effect on the dynamics as increasing the cost $k$ by $\tau(\beta-\alpha) \pi$. This implies in particular that increasing the punishment $\pi$ or the testing probability $\tau$ or the accuracy of the test $(\beta-\alpha)$ may actually lead to more doping in some cases.

We have introduced testing and punishments in a particularly simple fashion here. A possible variation is to assume that the losing party inherits the prize if the losing party passes the doping test but the winning party does not. The dynamics of this case (a static version of this situation is the subject of BERENTSEN [2002]) would be quantitatively different from our model, but the main qualitative features would probably remain: there are equilibria in which the high-ability players are more likely to cheat, and there can be Lyapunov-stable cycles.

\subsection{Three Population Games}

So far we have restricted attention to games with only two types of players. But the logic of doping games is not restricted to this case, and the analysis can be extended to a richer selection of types. A doping game with three types of players, for instance, would feature a highly talented population, an intermediately talented population, and a weakly talented population, with given population shares $q_{1}, q_{2}$, and $q_{3}:=1-q_{1}-q_{2}$. Two randomly chosen players from the whole population play the contest. The winning probabilities are influenced by the talent of the players and by whether they use doping or not.

Such a game has many parameters: the population shares, the cost of doping, and the winning probabilities of the different types of agents in all possible matches. Essentially, nothing changes with respect to the two-population doping game. For some parameters we find almost globally stable strategies: stationary points on the boundary of the strategy space. For some parameters, there are stationary points that are interior for two player types (meaning that a portion of players of this type stay clean while others dope), but the players that belong to the third type all do the same (all dope or all stay clean). And for some parameters, there are stationary points where all three player types end up in the interior of the strategy space, indicating that for each type, some dope and some do not.

Moreover, one can also generate Lyapunov-stable cyclical orbits around such interior stationary points. As an illustration, Figure 3 depicts such an orbit. This example assumes equal population shares $q_{1}=q_{2}=q_{3}=1 / 3$, and cost of doping $k=1 / 3$. Doping has the effect of allowing a weak player to win for sure against 
Figure 3

Cycles in Three-Population Doping Games

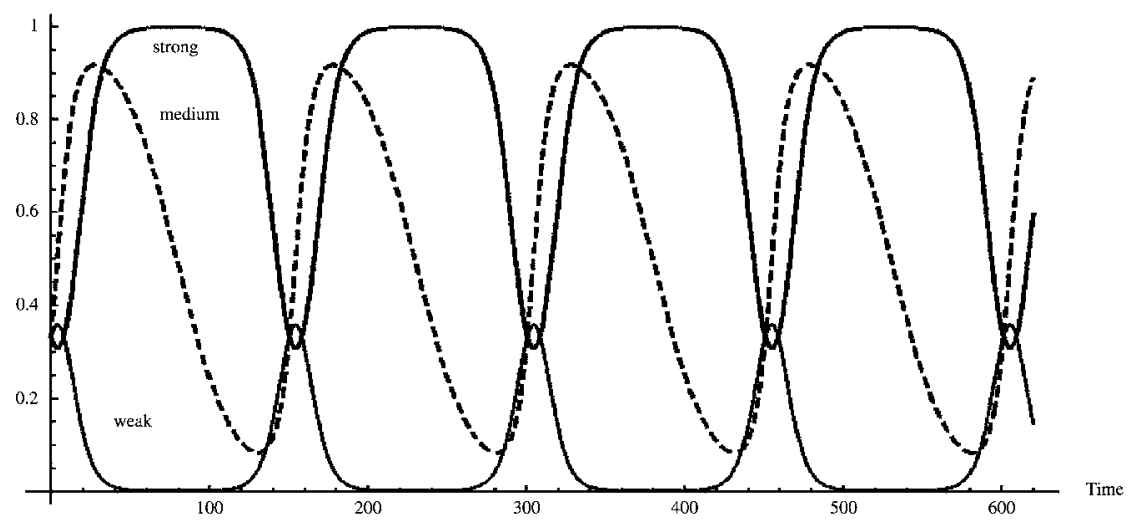

a clean mid-talent type, but not against a clean high type. A doped mid-talent type wins with certainty against a clean high type. In this example, most of the time almost all highly talented players use doping, while weakly talented players almost never use doping. The fraction of mid-talented players that dope is initially large but decreases as long as the fraction of strong players that use doping is large. When the fraction of mid-talented doped players has decreased enough, the benefit of doping for weak players increases, because with doping they have a chance to win if they meet a clean mid-talented person. Thus, the weak players begin to use doping. For the highly talented people, the benefit of doping becomes smaller, however, because so few mid-talented people use doping. Hence, the share of high types that use doping reduces rapidly. This, however, incites mid-talented players to resume doping, and we are back to square one.

\section{Conclusion}

In this paper we have analyzed an evolutionary game between two athlete types who can use performance-enhancing drugs at a cost. We find that, depending on parameter values, different asymptotically stable strategy equilibria exist on the boundary of the strategy space, or if not, then an interior strategy equilibrium exists, which is only weakly (Lyapunov, but not asymptotically) stable. In that case, there are also cyclical orbits, where doping and staying clean alternate. The reason for this behavior is that the two-population game has a structure similar to the matchingpennies game, unlike the one-population doping game, which has the structure of the prisoners' dilemma. 
Although we couch the discussion of the model in terms of sports, the model does have applications outside of sports. The strategic incentives of a manager or firm to misreport profit is one example. With this interpretation, our model suggests that with some parameter combinations, high-ability managers may be more likely to cheat. Also, we may observe waves of cheating and honesty.

\section{References}

BERENTSEN, A. [2002], “The Economics of Doping,” European Journal of Political Economy, $18,109-127$.

- -, E. BRÜGGER, AND S. LÖRTSCHER [2003], “On Cheating and Whistleblowing,” Discussion Paper, University of Bern.

Hofbauer, J., And K. Sigmund [1998], Evolutionary Games and Population Dynamics, Cambridge University Press: Cambridge.

KAndori, M., G. MAILATH, AND R. RoB [1993], "Learning, Mutation, and Long-Run Equilibria in Games," Econometrica, 61, 29-56.

LAZEAR, E. P., AND S. ROSEN [1981], "Rank-Order Tournaments as Optimum Labor Contracts," Journal of Political Economy, 89, 841-864.

TAYLOR, P., AND L. JONKER [1978], "Evolutionary Stable Strategies and Game Dynamics," Mathematical Biosciences, 40, 145-156.

WeIBULL, J. [1995], Evolutionary Game Theory, MIT Press: Cambridge, MA.

Aleksander Berentsen

Yvan Lengwiler

Department of Economics (WWZ)

University of Basel

Petersgraben 51

4003 Basel

Switzerland

E-mail:

aleksander.berentsen@unibas.ch

yvan.lengwiler@unibas.ch 